Appl. Set-Valued Anal. Optim. 2 (2020), No. 2, pp. 139-141

Available online at http://asvao.biemdas.com

https://doi.org/10.23952/asvao.2.2020.2.01

\title{
EDITORIAL \\ A SPECIAL ISSUE DEDICATED TO HONG-KUN XU ON THE OCCASION OF HIS 6OTH BIRTHDAY, PART I
}

Professor Hong-Kun $\mathrm{Xu}$ is an outstanding mathematician and a leading expert in the theory of fixed points of nonlinear operators. This Special Issue on Trends in Fixed Points of Nonlinear Operators is dedicated to him on the occasion of his 60th birthday.

Professor Hong-Kun Xu was born in July, 1960, in Zhejiang Province, China. He received his Bachelor's degree from Zhejiang Normal University in 1982, Master's degree from Zhejiang University in 1985, and Ph.D. degree from Xi' an Jiaotong University in 1988. He then started his career as Lecturer at East China University of Science and Technology. Now, he is a distinguished professor at Hangzhou Dianzi University. Before moving to Hangzhou, he worked at several famous universities around the world. Professor Hong-Kun Xu has produced dozens of Master's and Ph.D. students, most of them are now active experienced researchers in the mathematical community. He served as a member of the editorial boards of several international journals, including Nonlinear Analysis: Theory, Methods and Applications, Carpathian Journal of Mathematics, Optimization, Fixed Point Theory, Journal of Nonlinear and Convex Analysis, and Journal of Nonlinear and Variational Analysis, etc.

Professor Hong-Kun's main scholarly contributions are in fixed points of nonlinear operators. In the early stage of his career, he worked mainly on iterative methods for fixed points of nonexpansive mappings, Banach space geometry, and multivalued mappings. He then moved to the study of optimization problems and their real applications. Up to now, he has published, according to MathSciNet, more than 200 papers in peer-reviewed international journals and he was selected into the list of Highly Cited Researchers by Thomson Reuters/Clarivate Analytics and Elsevier In addition, he was also elected to a member of the Academy of Science of South Africa (2005), and a fellow to the World Academy of Sciences (2012).

This special issue aims to acknowledge and celebrate his contributions to the theory of fixed points. Part I of the special issue contains eight papers contributed by leading experts as follows:

T. Domínguez Benavides and P. Lorenzo Ramírez, in their paper "Fixed points for multivalued non-expansive mappings", revise some fixed point results for multivalued non-expansive mappings in Banach spaces and modular spaces, and state some new results depending either on the Opial modulus and or on the Partington modulus in modular spaces.

Based on a very recent work by R. T. Rockafellar and the Yosida approximation, Abdellatif Moudafi and Noureddine Lehdili, in their paper "From progressive decoupling of linkages in

(C)2020 Applied Set-Valued Analysis and Optimizaton 
variational inequalities to fixed-point problems," propose a regularization approach of the idea and develop a similar analysis for fixed-point problems. They introduce various elicitation notions, and present the related progressive decoupling algorithms. Their convergence properties are investigated under global elicitability assumptions and an application to decomposition and splitting problems is also briefly mentioned.

In the paper "Fixed point results for multi-valued locally contractive operators," Adrian Petruşel and Gabriela Petruşel present several fixed point results for multi-valued $(\varepsilon, \alpha)$-uniformly locally contractive operators in complete $\varepsilon$-chainable metric spaces. Connections to the mathematics of multi self-similar sets are given.

Jong Soo Jung, in the article "A general iterative algorithm for generalized split feasibility and fixed point problems," introduces a general iterative algorithm based on the hybrid steepest descent method for finding a common element of the solution set of generalized split feasibility problem and the fixed point set of a continuous pseudocontractive mapping. He also establishes the strong convergence of the proposed iterative algorithm and finds a minimum-norm element in the common set of the two sets in a Hilbert space.

The primary focus of the paper, "Strong convergence theorems of a split common null point problem and a fixed point problem in Hilbert spaces" by Minh Tuyen Truong, Thi Thu Thuy Nguyen and Minh Trang Nguyen, is to introduce a new iterative method for finding a common solution of the split common null point problem and the fixed point problem in Hilbert spaces. They obtain the strong convergence of the new iterative method and also give some applications and numerical experiments to support their main convergence results.

Habtu Zegeye and Oganeditse A. Boikanyo, in the paper "A general split fixed point problem governed by demicontractive mappings in Banach spaces," proposes an iterative process, which converges strongly to the solution of a general split fixed point problem governed by demicontractive mappings, and prove strong convergence theorems in Banach spaces.

Laleh Eslamizadeh and Eskandar Naraghirad, in their work "Bregman common skew-attractive point theorems for semigroups of nonlinear mappings in Banach spaces" prove Bregman common attractive point and Bregman common skew-attractive point theorems for a semitopological semigroup $S$ of Bregman type nonexpansive (not necessarily continuous) mappings acting on a closed convex subset $C$ of a Banach space $E$.

The goal of the work "A shrinking projection algorithm for proximal split feasibility and fixed point problems" by Jinzuo Chen is to study proximal split feasibility, and fixed point problems. A shrinking projection algorithm is introduced in the framework of Hilbert spaces. It is proven that the sequence generated by the proposed iterative algorithm converge to a common solution of a proximal split feasibility problem and a fixed point problem of an asymptotically $k$-strictly pseudo-contractive mapping in the intermediate sense.

Finally, we express our most sincere gratitude to all the authors who have contributed to this special issue and to the reviewers who helped us with their thorough reviews.

Adrian Petruşel

Department of Mathematics

Babeş-Bolyai University Cluj-Napoca, Romania E-mail address: petrusel@math.ubbcluj.ro 


\section{Xiaolong Qin Department of Mathematics} Hangzhou Normal University, Hangzhou, China E-mail address: qxlxajh@163.com 